\title{
Psychotherapy: advances in training methods
}

\author{
Frank Margison
}

Trainees need to gain experience in the psychotherapies, but practical problems in implementing training programmes have made it difficult for many trainees to access the full range of experience.

This paper focuses on practical methods of training that do not require tutors to be experts in psychotherapy. Examples are given of integrating the various methods of teaching psychotherapy within a local training programme. Teaching basic psychotherapy skills does not rely on any particular theoretical perspective, but uses educational principles to help trainees to monitor their own role in the interview, and to have strategies to deal with difficult interactions.

Box 1. Examples of educational objectives in interview training

To be able to:

Gather information

Clarify information

Make diagnostic interventions to elicit signs and symptoms

Agree a formulation

Agree a treatment plan

Check progress

Review aims of treatment

Deal with resistance or hostility

Manage the patient's feelings of anger, sadness, fear and suspicion

Recognise and repair problems in the therapeutic alliance

\section{Summary of requirements for training}

\section{Introductory training}

In the first year of experience in psychiatry, there are two main aspects to psychotherapy training: interview skills training and a case discussion group, in which trainees discuss the psychotherapeutic aspects of their day-to-day work. Some possible learning objectives of these experiences are given in Box 1.

\section{Pre-membership supervised experience}

After the introductory experience, trainees should take on supervised cases. There should be a minimum of one long case and two brief cases in each of the two main modalities studied: cognitive-behavioural and psychodynamic-interpersonal therapy. Where possible, these experiences should be supplemented by experience in systemic approaches (with couples and families) and group methods of treatment. It is not required that these cases come from specialist psychotherapy departments. On the contrary, all psychiatric specialities can provide examples of relevant training opportunities - a few examples are shown in Box 2.

Some psychotherapeutic approaches are relevant across all specialities. Issues like managing

Frank Margison has been a consultant psychotherapist in Manchester since 1983 (Gaskell Psychotherapy Centre, Manchester Royal Infirmary, Swinton Grove, Manchester M13 OEU) and prior to that he was Clinical Lecturer at the University of Manchester, where he was involved in developing teaching methods for psychodynamic-interpersonal therapy using Hobson's Conversational Model. He was Programme Director for Psychotherapy in the North West Deanery until recently, when he took over as Chair of the Psychiatry Specialist Training Committee. His research interests are in measuring psychotherapy outcomes and in developing and evaluating teaching methods in psychotherapy. 
Box 2. Some examples of training opportunities by speciality

General adult psychiatry

Ward groups in in-patient settings (e.g. community groups, expressive art groups) (group therapy or other modality)

\section{Rehabilitation psychiatry}

Social skills training (cognitive-behavioural therapy)

\section{Substance misuse}

Motivational interviewing (cognitive-behavioural therapy)

\section{Liaison psychiatry}

Dealing with somatisation of distress (psychodynamic-interpersonal or cognitive-behavioural therapy)

Dealing with effects on body image of surgical interventions (psychodynamic-interpersonal or cognitive-behavioural therapy)

\section{Old age psychiatry}

Reality orientation (other modality)

Family interventions to minimise conflict (systemic therapy)

\section{Child psychiatry}

Drawing-up genograms (systemic therapy)

Family assessment and intervention (systemic therapy)

Parenting skills enhancement (cognitive-behavioural therapy)

Forensic psychiatry

Cognitive-behavioural strategies to reduce violence and re-offending (cognitive-behavioural therapy)

Self-monitoring of the arousal cycle in sex offenders (cognitive-behavioural therapy)

Psychiatry of learning disability

Behavioural interventions to reduce self-harm or challenging behaviours (cognitive-behavioural therapy)

self-harm, loss and bereavement can be considered as generic skills and can be learned in any setting. Examples of these are given in Box 3.

\section{Post-membership psychotherapy experience}

Specialist registrars (SpRs) need to train in aspects of psychotherapy that are relevant to their particular speciality. A common request is for training in brief, focused methods of therapy relevant to that speciality.
The examples given in Boxes 2 and 3 in the context of senior house officer training, however, are often still relevant at SpR level, and educational supervisors can agree educational objectives appropriate for future practice as specialists from these examples.

\section{Continuing professional development in psychotherapy}

The skills described above are relevant also for careergrade psychiatrists in all specialities (Temple, 1999).

Box 3. Training opportunities: examples of generic skills relevant to all specialities

Specific cognitive-behavioural interventions for mood disorders, eating problems and positive symptoms of psychosis

Cognitive-analytic or psychodynamic skills to deal with recurrent relationship conflicts

Increasing compliance and reducing relapse rates

Reducing self-harm

Managing loss and bereavement 
With recent advances in training methods, it is not necessary to train to specialist level in these modes of therapy in order to assimilate the relevant clinical methods into routine practice. Continuing professional development workshops should build on existing skills rather than developing entirely new approaches.

Continuing professional development for consultants involves two distinct components. First, consultants may decide to enhance their own clinical practice in psychotherapy as an aim of continuing professional development. Second, the consultant may want to develop his or her educational skills to enhance the practice of their trainees.

The remainder of this article deals with developing educational skills and overcoming some of the practical difficulties.

\section{Practical issues}

Pressures of other training requirements and the lack of supervisors have made local tutors feel that the current psychotherapy training requirements are difficult to implement.

Without minimising the logistical difficulties, there are ways of re-construing current training opportunities to allow trainees to gain relevant experience. Some examples are given in Box 2 . For a training scheme as a whole, these opportunities can be collated so that specific opportunities to enhance psychotherapeutic skills are available in most, if not all, placements.

Adding elements of experience in an uncoordinated way leads to piecemeal training. So, a log book overseen by the tutor should be used to co-ordinate experience. The tutor can prompt trainees about how the current or next post might be used to gain relevant experience. If educational supervisors are to feel confident in developing psychotherapy training opportunities in their own work setting, they may themselves need to practise some of the newer educational approaches described later. Such experiences are becoming increasingly available in continuing professional developmen workshops, whether organised locally or at specialist centres.

Even when College tutors collate all the additional training opportunities, there is still a shortage of supervisors for cognitive-behavioural therapy and psychodynamic-interpersonal methods in many areas. To minimise this difficulty, it may be necessary to concentrate teaching and supervisory resources on key objectives. This might mean that case discussion groups and interview skills training are organised by non-specialists, and specialist input is concentrated on developing defined, measurable therapeutic competence in each of the main modalities.
A log book that simply records the number of hours of experience, without defining clear learning objectives and skills, is inadequate for the task of developing psychotherapeutic competence (a theme to be developed in a future paper). The remainder of this paper deals with how a non-specialist can help trainees develop the foundations for safe and effective psychotherapeutic practice.

Psychotherapy training draws on educational principles relevant to adult learning. In particular, the trainer is using educational principles of : (a) enhancing trainees' ability to monitor their own practice; (b) giving timely, accurate and constructive feedback; and (c) using knowledge of group dynamics to increase learning.

\section{Developing basic therapeutic skills}

\section{Interview training}

In good interviewing, the trainee knows what is being done, how it sounds, why a particular intervention is being made, and understands the basic purpose of the interview. This implies that a trainee is developing a repertoire of interview approaches to achieve some of the objectives stated in Box 1.

\section{Listening to tapes}

Interview skills training can use recordings of sessions with patients, or role-play (see below).

Tapes of sessions are a practical way of allowing trainees to process the material in their own time, or to listen in detail with a trainer - but ethical issues about confidentiality need to be considered. Agreement with the patient is needed about the purpose of taping the session. Normally the tape is erased after the trainee has listened to the tape, and if it is to be kept any longer, written consent is needed.

The two techniques used most often to develop basic interview skills are listening with a trainer and using structured methods to describe sessions.

The initial objective is to be able to recognise which interventions are being used (what). Once this has been achieved, there can be a discussion of how and why to bring out trainees' aims at that moment, and also for the interview as a whole. Once these learning objectives have been met, discussion can take place about alternative interview strategies and what can make an interview difficult.

Ownership of the tape is crucial. Trainees need to determine when and how it is played. They often 
feel on the spot and judged unless they feel they can bring the tape for assistance rather than scrutiny. Once they feel more confident, it may be possible to play random extracts, and it is remarkable how often the key themes of the whole session are condensed into each brief extract. The most effective way of giving feedback should be in the form "Isn't it interesting that you talk over the patient when she seems upset", rather than an instruction to behave differently.

This actually mirrors the collaborative approach that good therapists use. If feedback is to change behaviour, it is most effective when it helps trainees to assimilate new information. A spirit of mutual enquiry is encouraged, helping trainees to notice the effect of interventions rather than following a rule book.

Keen trainees can also be encouraged to listen to their tapes systematically, for example listening for metaphors, for points where trainee and patient talk simultaneously, for similarities and differences in tone of voice, or for how the depth of the experience varies through the session.

These techniques are relevant whether the tape is of a routine out-patient clinic, a discussion about drug treatment or a discussion about leave. It may be particularly relevant when trainees are carrying out a course of psychological treatment, but the use of tapes should not be restricted to specialist practice. Although the tape is there primarily to assist trainees in gaining experience, it is also a method of quality control so that the consultant has a clearer idea of what is happening in sessions. Trainees can simply avoid bringing awkward bits of a session where they felt their intervention had been less than perfect-most experienced trainers suspect that the trainee who never brings along clumsy or failed interventions should be a cause for concern.

\section{Helping trainees to analyse their sessions}

Cobb \& Lieberman (1987) suggested a systematic way of developing the capacity to listen to sessions. Their 'interactogram' model described three levels of analysis of an interview. Their first level of training deals with what was happening in terms of different types of questions, facilitation, statements and other interventions, and then involves listening to the impact on the conversation.

This approach is based on micro-skills analyis following the work of Ivey \& Simek-Downing (1980) with counsellors, Maguire et al (1978) with medical students, and Hobson and colleagues (Hobson \& Margison, 1983; Goldberg et al, 1984) using the 'conversational model' of therapy with psychiatry trainees.

The second level of the interactogram brings in the intention (asking why a particular intervention was used) and a more detailed analysis of the impact on the patient. Finally, the third level of the interactogram draws on concepts of transference and the therapist's response - the countertransference. This brings in higher level skills in understanding the relationship, and trainees' underlying hypotheses are made explicit.

\section{Using role-play}

Trainees are reluctant to use role-play when they feel they might be exposed or humiliated. The atmosphere needs to be serious but still lighthearted. There should be a steady increase in the level of complexity of the role-plays. An example is given below based on the training offered at the Gaskell Psychotherapy Centre in Manchester.

The training starts with the basic assumption that trainees worry that they will not be able to cope with difficult situations. Kagan (1980) developed a training method focused on these therapist fears. He suggested that therapists typically "feign clinical naiveté" with a supervisor to avoid possible humiliation about their basic fears. He describes these as:

- a fear of being engulfed or engulfing (seducing) the other; and

- a fear of being attacked or attacking the other.

These are the basic fears in any interpersonal interaction. The role-plays set up a series of escalating difficulties to desensitise trainees to their fears and to provide an appropriate therapeutic response when patients re-enact their problematic relationship in the therapeutic setting.

A standard format is used for all the role-plays. The group uses two chairs for the members playing 'therapist' and 'client'. The client is asked to prepare a couple of sentences summarising the basic information about age, gender and what the main problems are. The client or the therapist (or occasionally both) is given cue cards which have a brief instruction explaining how the interview should be approached. The role-plays run for about five minutes and then the tutor asks, in turn, the role-players and then the rest of the group for comments, having set ground rules about commenting on what has been seen, avoiding personal criticism, suggesting alternatives and making links to other clinical work.

\section{Level 1: Basic skills}

These use simple instructions, such as "avoid eye contact", or "lean forwards".

The aim is to familiarise the trainees and to begin focusing on the emotional impact of different behaviours. 


\section{Level 2: Negotiation}

The cards deal with the emotional impact of changing the assumed contract. For example, the client might ask to meet more frequently, or the therapist might need to change the session time.

The aim is to increase trainees' familiarity with the emotional subtext of a session.

\section{Level 3: Dual experience}

These cards develop the ability to explore difficult feelings in a session using deliberate prompts. For example, both patient and therapist are told not to take what the other person says at face value, or one participant is asked to comment on how the other is dressed or their appearance.

The aim is to develop understanding of how easily persecutory feelings can be established when either party neglects the need for personal space.

\section{Level 4: Dilemma cards}

After three weeks on the previous tasks, trainees then use role-plays that desensitise them to Kagan's therapeutic nightmares. These include situations that suggest possible boundary threats (e.g. the suggestion of meeting elsewhere), the possibility that feelings might get out of control (e.g. mention of suicide or smashing the room up), or personal, hostile comments to the therapist.

The aim of these latter role-plays is to become familiar with the powerful feelings in a difficult session, and to give trainees a framework within which they can understand what is happening.

Commonly, trainees have had bad previous experience of role-plays where they have felt humiliated or exposed. It is important for the teacher to establish an atmosphere that allows in-depth exploration for the role-players and the group as a whole. The structure of the session prevents the drift from being a training group to a quasi-therapeutic group.

\section{Setting up a productive case discussion group}

The case discussion group is not the exclusive preserve of specialist psychotherapists, as many of the teaching skills are present across speciality boundaries. Where the specialist might have a particular advantage is in drawing up a psychodynamic, cognitive-behavioural or systemic formulation, but these skills are relevant at a later stage in training.

Combining case discussion with reading relevant papers is a useful introduction to learning psychotherapeutic skills. A regular group of about 6-8 trainees, meeting in part of 'protected' educational time for about 75 minutes, is the usual format. Trainees bring cases that they have found difficult. Cases should be difficult not because of the technical issues of choice of medication, or diagnosis, as these should be discussed elsewhere, but because trainees find the interaction difficult in some way. Trainees should bring clinical situations which they find challenging, such as a patient asking a personal question, making threats or making the trainee feel incompetent.

This learning experience focuses on effective communication, whereas personal therapy has quite different aims. The trainer needs to preserve a boundary so that trainees keep to the task of understanding their communication with patients. The trainer may need to be directive in steering the discussion away from accounts of trainees' personal experiences of, say, relationship breakdown. There may well be a place for this degree of personal exploration, particularly for specialist psychotherapists, but it is distinct from the basic skills taught in the first year of training.

\section{Conceptualising difficulties in the therapeutic interaction}

Davis et al (1987) developed a way of systematically exploring trainees' difficulties. They produced a useful check-list of therapist difficulties (see Box 4) and also of coping strategies (see Box 5). Trainees can use these to recognise the sort of interpersonal and professional pressures they are facing. The list can be used systematically to understand difficult situations in a research context, but here it is recommended simply to explore which of the experiences they recognise and also to distinguish their adaptive from maladaptive coping responses.

The trainer's task is not to solve the problem brought by trainees, but gradually to get the group to recognise that these experiences are universal and often of diagnostic and therapeutic importance.

\section{Case example}

A 26-year-old male trainee was dealing with an angry 46-year-old female patient whose son had died in a motor cycle accident. The son had been planning to leave home to live with his girlfriend who was pregnant, and the mother had dealt only partially with her religious beliefs about the son not being married. She asked the trainee whether he was Roman Catholic and whether he thought it was a sin to commit suicide. 


\section{Box 4. Difficult experiences}

\section{Trainee feels \\ Incompetent \\ Damaging \\ Puzzled \\ Threatened \\ Out of rapport \\ Stuck \\ Thwarted \\ Personal issues evoked \\ Facing painful reality of the situation \\ Facing ethical dilemma}

The discussion covered several topics of relevance to the trainee's difficulties including:

\section{Dealing with an angry patient}

The group discussed the fear that the patient's feelings of sadness or anger might become uncontrollable if her defences were challenged, and the trainee's embarrassment when the patient could be heard shouting at him on the corridor. Some strategies to help contain the situation emerged.

\section{Dealing with personal questions}

The group dealt with the fine balance between declining to answer and the need to keep open an exploration of why she asked. In this case we speculated that she was seeking reassurance that her feelings of anger and blame towards the girlfriend were partly understandable in terms of her religious beliefs, and reassurance that there is some meaning in death. We also aimed to open a discussion about her thoughts of suicide.

\section{Dealing with the trainee's own feelings}

Using the check-list we each tried to imagine how he might feel. Common responses, which he shared, were of feeling incompetent, puzzled and threatened, as well as aware of the various ethical dilemmas and the painful reality the patient faced. The trainee was also able to acknowledge that the situation brought up echoes of his own relationship with his mother. We agreed that this was worth keeping in mind, but should not be explored further in the group.

Over the first year of training, each trainee learns to recognise his or her characteristic response to different pressures, and to what degree such feelings are typical enactments of the patient's characteristic relationship patterns. The repertoire of interview skills and strategies learned in the first stage of

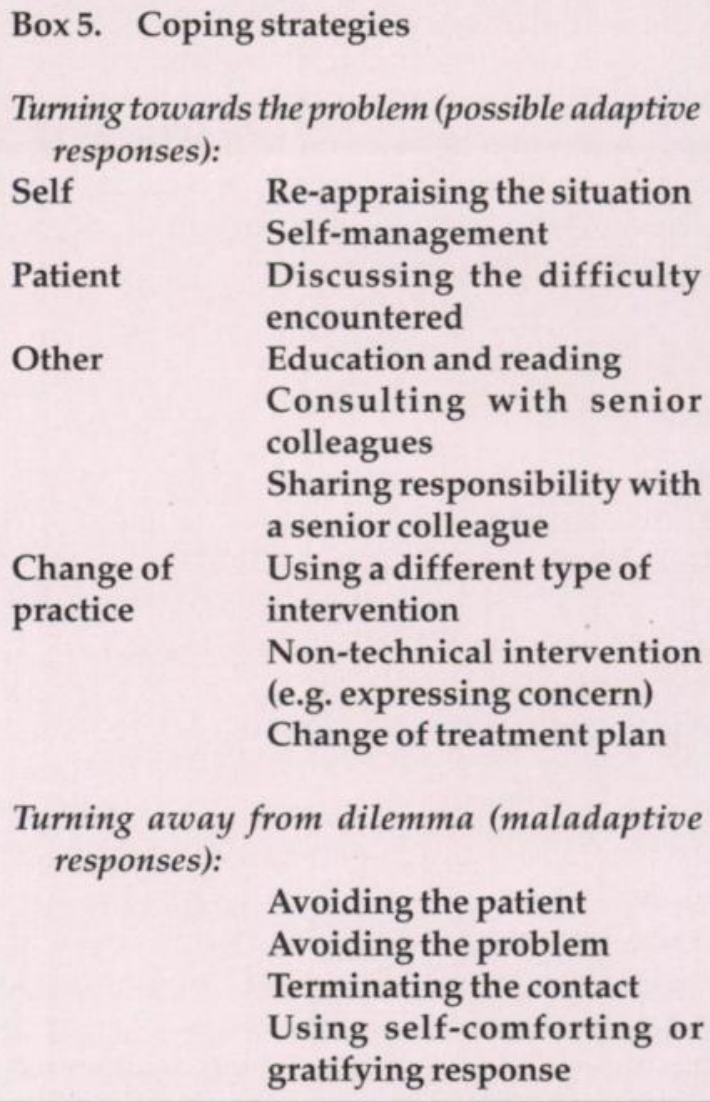

training is relevant in defusing difficult situations, and in making even difficult interviews productive. More detailed formulation of the relationship patterns and enactments are part of the case supervision that comes later in training, as part of supervised practice.

\section{Other basic skills at the beginning of training}

During the early part of training there are some other basic skills to learn before starting supervised clinical work. Three are genograms, narrative history-taking and process records of sessions.

\section{Drawing-up genograms}

A genogram draws together in a diagrammatic form the members of a patient's family and deaths, separations and losses, along with brief "pen pictures" of the key family members (McGoldrick \& Gerson, 1985). The genogram incorporates a number of conventions in drawing-up a family tree, some of 
which are familiar from genetic family trees (squares for males, circles for females, etc.) and some of which are more descriptive. For example, double lines are used for particularly close ties and jagged lines for conflictual relationships (see McLeod, 1998). The genogram is often constructed in a family session, but may also be used to bring a systemic perspective in individual therapy.

\section{Narrative history-taking}

Roberts (1997) has described how the underlying narrative structure of a patient's experience can be detected during a conversation. Contrary to popular myth, this narrative structure is present even in the presence of acute psychosis, and the underlying interpersonal fears and concerns will be expressed obliquely in the discussion of delusional or hallucinatory material. Trainees need to grasp the differences between trying to follow the underlying narrative story, identifying psychopathology and attributing causality.

A session also has a narrative structure - simply, the session is divided like chapters of a book into a meaningful structure. There is no ultimate test of correctness of such a structure, but trainees learn that there are naturally occurring transitions that can be expressed in terms of emerging meaning. Trainees are encouraged to develop their own idiosyncratic style, which is likely to capture the essence of the session better than a restrictive system. For example, the session described earlier might have the chapter structure shown in Box 6 .

\section{Process notes}

Trainees often do not know how to write-up process notes. Although most professionals should be familiar with the need to keep a formal record of certain issues (such as risk assessment and communication with other agencies), process notes have a different function. Some trainees find it helpful to use a structured method of writing up process notes. They can prepare some standard record sheets divided up into the relevant sections shown in Box 7.

These process notes can be compiled every few sessions to summarise the main issues arising in the therapy.

\section{Summary}

This paper deals with methods of teaching psychotherapy from the perspective of a psychiatric tutor or educational supervisor. It gives a foundation for establishing in-house training in psychotherapy. This means that scarce training resources from specialist psychotherapists can be used to supervise trainees on direct clinical work in cognitivebehavioural and psychodynamic psychotherapy.

The range of methods helps trainees to gain basic psychotherapeutic skills. As part of the regular teaching programme, in protected time, there may be an interview skills group running for six months, followed by a case discussion group for the next six months, as part of a rolling programme.

In addition to group teaching, consider using some of the weekly hour of educational supervision for other training opportunities. You might encourage the construction of genograms as part of routine practice, or help trainees monitor the difficulty of an interview using the educational tools described above. Whatever the focus of the teaching, direct feedback helps trainees to assimilate the suggested changes in their interview style.

You can help trainees to integrate the varied educational experiences described in this paper

Box 6. Example of chapter structure of a session

Mrs Brown tells how the ambulance let her son die by the roadside

Mrs Brown is reminded how angry she feels

Mrs Brown sweeps on regardless and tells how she will make them pay

Dr Stephens feels uncomfortable thinking about the possible complaint that might come his way

Dr Stephens faces the anger and reflects that Mrs Brown might be angry with him

Mrs Brown says: how angry she is with lawyers and people who meddle in other people's lives; that lawyers are not reliable; and that her lawyer is never available

Dr Stephens draws parallel with times that he is not available

Therapist draws link with change of session time: changing session times has made her feel anxious before about him not being around for her

Mrs Brown goes back to her son being 'left by the roadside'

Dr Stephens tries to use metaphor of being 'left by the wayside' as a theme linking her experience in the session and after her son's death 
using the logbook and by setting psychotherapy educational objectives at the beginning of each attachment.

Wherever possible, link the training to directly observed material, whether from tapes or in role- play. Direct feedback about the interview style is more effective in changing behaviour than teaching abstract principles. The direct feedback can be assimilated best as part of a collaborative educational

Box 7. Systematic session recording

Name

Therapist

Session number

Date

Observation of client

Hypotheses based on observation of client

Observation of therapist

Hypotheses based on observation of therapist

Observation of interaction

Hypotheses based on observing interaction

Progress with core formulation

Progress with aims of therapy

Reminders for next session

Administrative

Themes to be monitored

Predictions

Other notes for supervision
Joan Brown

Michael Stephens

8 of 12

1 March 1999

Still very angry and cuts me off whenever I try to speak Still wearing long black coat

Angry with everyone because she feels abandoned and betrayed by son's death

Cannot allow another perspective (in case she becomes tearful and loses face)

Makes me think of her still being 'in mourning'

I keep trying to get a word in edgeways

I feel edgy and uncomfortable in the session; like I'm about to be criticised all the time

She is actively blocking any attempt I make to express feelings

I am being put on the defensive, and carrying the blame

Very edgy, talking over each other, both talking in abstractions

Active blocking of feelings

Re-enacting 'someone has to be to blamed' for how I feel

Further information supporting 'blocked grieving' - cannot accept her anger towards him for abandoning her (twice: girlfriend and his death)

Therapy seems blocked: no opportunity for reflection or expression of feelings. Caught in a re-enactment of how she was with her son?

Remind her how many sessions left, and that session time in two weeks has to be changed

Unacknowledged anger towards son for abandoning her

Changing session time will be a possible focus for anger towards me, but probably difficult to explore

Start tape at \#126 where she is talking about making a complaint to the ambulance service for not responding quickly enough (a disguised reference to session)

NB The text on the left shows the pro forma and the text on the right shows the elements to be filled in by the trainee after the session 
approach using principles of effective group working, or of effective educational supervision.

To encourage desirable aspects of interview style the trainer needs to be aware of the sequence of recognising, practising and eventually mastering a new skill. Where there are aspects of the interview leading to difficulty, look at the possible reasons in a structured way before providing alternative strategies.

The role of the non-specialist teacher is commonly understated in helping trainees to acquire psychotherapy skills.

\section{References}

Cobb, J. P. \& Liebermann, S. (1987) The grammar of psychotherapy. A descriptive account. British Journal of Psychiatry, 151, 589-594.

Davis, J. D., Elliott, R., Davis, M. L., et al (1987) The development of a taxonomy of therapist difficulties. British Journal of Medical Psychology, 60, 109-120.

Goldberg, D. P., Hobson, R. F., Maguire, G. P., et al (1984) The clarification and assessment of a method of psychotherapy. British Journal of Psychiatry, 144, 567-580.

Hobson, R. F. \& Margison, F. (1983) A Conversational Model of Psychotherapy: A Teaching Method (set of videotapes and handbook). London: Tavistock.

Ivey, A. E. \& Simek-Downing, L. (1980) Counseling and Psychotherapy: Skills, Theories and Practice. New Jersey: Prentice Hall.

Kagan, N. (1980) Influencing human interaction - eighteen years with IPR. In Psychotherapy Supervision: Theory, Research and Practice (ed. A. K. Hess). New York: John Wiley.

McLeod, J. (1998) An Introduction to Counselling, pp. 118121. Buckingham: Open University Press.

McGoldrick, M. \& Gerson, R. (1985) Genograms in Family Assessment. New York: Norton.

Margison, F. (1991) Learning to listen: teaching and supervising basic psychotherapeutic skills. In Textbook of Psychotherapy in Psychiatric Practice (ed. J. Holmes), pp. 165-186. London: Churchill Livingstone.

Maguire, P., Goldberg, D., Hyde, C., et al (1978) The value of feedback in teaching interviewing skills to medical students. Psychological Medicine, 8, 695-704.

Roberts, G. A. (1997) Meaning and madness. A narrative approach to psychopathology and treatment. In Psychotherapy of Psychosis (eds C. Mace \& F. Margison), pp. 255-271. London: Gaskell.

Ryle, A. (1990) Cognitive Analytic Therapy: Active Participation in Change. London: Wiley.

Temple, N. (1999) Should consultant psychiatrists be trained in psychotherapy? Advances in Psychiatric Treatment, 5, 288295.

\section{Multiple choice questions}

1. Teachers of interviewing skills to psychiatry trainees should:

a be registered specialists in at least one modality of psychotherapy

b focus primarily on the detection of psychopathology c be familiar with the principles of feedback to alter behaviour

d use an exclusively psychodynamic focus

e use knowledge of group dynamics to increase learning.

2. Case discussion groups:

a rely exclusively on the work of Michael Balint

b should have fewer than four trainees

c provide a useful experience of therapy for trainee psychiatrists

d give feedback about the impact of the trainee's conversational style

e are often used to discuss difficult experiences when on-call.

3. Analysis of therapist difficulties in a case discussion group:

a relies mainly on self-reflection by the therapist reporting the case

b commonly provides discussion of ethical dilemmas arising in practice

c typically makes links with the trainee's early life experience

d commonly uses a check-list method to clarify the nature of the difficulties

e uses a classification based on the type of interview setting.
MCQ answers
12
a $\mathbf{F}$
3
b $\mathrm{F}$
F
b $\mathrm{F}$
c F
c $\mathrm{T}$
d $\mathrm{T}$
d $\mathbf{F}$
e $\mathrm{T}$

$\begin{array}{ll}\text { a } & \text { F } \\ \text { b } & \text { T } \\ \text { c } & \text { F } \\ \text { d } & \text { T } \\ \text { e } & \text { F }\end{array}$

